\title{
Multidrug-resistant tuberculosis: eight years of surveillance in France
}

\author{
J. Robert, D. Trystram, C. Truffot-Pernot, V. Jarlier
}

Multidrug-resistant tuberculosis: eight years of surveillance in France. J. Robert, D. Trystram, C. Truffot-Pernot, V. Jarlier. (C) ERS Journals Ltd 2003.

ABSTRACT: The aim of this study was to evaluate the annual prevalence of multidrugresistant tuberculosis (MDRTB) and to describe the characteristics of the patients with MDRTB in France.

Annual questionnaire surveys from 1992-1999 were mailed to all French microbiological laboratories performing mycobacterial cultures. A total of 264 distinct patients were reported to the National Reference Centre for Resistance of Mycobacteria to Antituberculosis Drugs during the 8-yr surveillance period resulting in a mean annual prevalence of MDRTB of $0.6 \%$.

A mean of $16 \%$ of the MDRTB patients were reported over several subsequent years. The majority of patients were male $(69.7 \%)$, foreign-born $(55.7 \%)$, with a previous history of treatment $(65.9 \%)$, and pulmonary involvement $(92.8 \%)$ with smear-positive results $(59.1 \%)$. Human immunodeficiency virus (HIV) coinfection was present in $20.8 \%$ of the patients. Strains were resistant only to isoniazid and rifampin in $37.9 \%$ of the cases, and additional resistance to both streptomycin and ethambutol was present in $\mathbf{2 5 . 8} \%$. HIV coinfection and female status were statistically associated with primary resistance, whereas smear-positive results were associated with secondary resistance. Foreign-birth and smear-positive results were associated with a chronic status.

The prevalence of multidrug-resistant tuberculosis is low in France $(<1 \%)$. However, a substantial proportion of patients remain positive for several years, suggesting nonoptimal management. Therefore, as recommended by the World Health Organization, a few reference teams, working in collaboration with national associations of physicians and microbiologists, should be established to improve the outcome of multidrug-resistant tuberculosis.

Eur Respir J 2003; 22: 833-837.
National Reference Centre for Antimicrobial Resistance of Mycobacteria, and BacteriologyHygiene Pitié-Salpêtrière Medical School, Paris, France.

Correspondence: J. Robert

Laboratoire de Bactériologie

Faculté de Médecine Pitié-Salpêtrière

91 Bd de l'hôpital

75634 Paris Cedex

France

Fax: 33145827577

E-mail: jrobert@chups.jussieu.fr

Keywords: Human immunodeficiency virus multidrug-resistant

surveillance

tuberculosis

Received: February 72003

Accepted after revision: June 242003
Acquisition of resistance to antituberculosis agents in Mycobacterium tuberculosis was described soon after their clinical use. In 1999, the prevalence of resistance to any of the first-line drugs (rifampin (RIF), isoniazid (INH), ethambutol (EMB) and streptomycin (SM)) reached $>30 \%$ among new cases of tuberculosis (TB) in several countries (Estonia, Thailand), and $>40-50 \%$ among previously treated cases (China, Estonia, Italy) [1]. Multidrug-resistant tuberculosis (MDRTB), defined as resistance of $M$. tuberculosis to at least INH and RIF, is especially worrisome because of its potential impact on the control of TB. Indeed, INH and RIF are the two major drugs in the treatment of $\mathrm{TB}$, and resistance to both of them and particularly to RIF represents a major risk factor for treatment failure [2, 3]. MDRTB has been reported in most parts of the world but it is more prevalent in countries where TB and human immunodeficiency virus (HIV) coinfection are endemic [4]. In the 1980s, because of a steady decline of the total number of cases, TB and especially MDRTB was abandoned as a public health priority in industrialised countries. TB became the focus of attention again in the early 1990s because of outbreaks of MDRTB in healthcare and correctional facilities in the USA, and because of a stop in the downward trend in TB prevalence in some industrialised countries [5-9].

In France, surveillance of drug resistance is not linked to the case notification system. National surveillance of TB drug resistance was discontinued in the early 1970s [10] but resumed in the 1990s. Surveillance of MDRTB was established in France at the national level in 1992 in order to evaluate the annual prevalence of MDRTB, and to describe the characteristics of patients with MDRTB. The present report gives the results of the surveillance for an 8-yr period, from the beginning of the programme in 1992, up to 1999.

\section{Methods}

\section{Definitions}

MDRTB was defined as resistance of $M$. tuberculosis to at least INH and RIF. According to previous history of treatment [11] at the time of MDRTB diagnosis, patients were classified into three groups: 1) previously untreated patients, or new patients, who had never taken any anti-TB drug or had taken anti-TB drugs for $<4$ weeks; 2) previously treated patients who had taken anti-TB drugs for $\geqslant 4$ weeks; and 3 ) unknown, all patients for whom the treatment history was unknown or doubtful. Chronic status was defined according to the World Health Organization (WHO). Foreign status was defined as place of birth.

Antimicrobial susceptibility tests to first-line drugs were initially performed by reporting laboratories. Confirmation of multidrug resistance and susceptibility tests to secondline drugs were performed on request by the National 
Reference Centre for Resistance of Mycobacteria to Antituberculosis Drugs (NRC-RAD) using the proportion method of Löwenstein-Jensen.

\section{Surveillance}

Surveys were conducted annually from 1992-1999 by standardised questionnaires sent by mail to a network of laboratories covering the entire French territory and including the hospital laboratories, Pasteur Institutes, and private laboratories performing mycobacteria cultures. These laboratories have been extracted from the national roster of all French laboratories performing medical analysis.

The total number of patients with culture yielding $M$. tuberculosis and, among them, the number of patients harbouring multidrug-resistant strains were recorded from each laboratory. For each patient with MDRTB, additional data were collected by mail or by phone by the microbiologist and the physician in charge of the patient. Data included age, sex, place of birth, site of infection, HIV coinfection, date of first diagnosis of MDRTB, smear result and susceptibility to first-line drugs. Duplicate reports were identified throughout the surveillance period by using initials of first and last name, date and place of birth. No additional information were recorded for non-MDRTB patients.

Data were entered into computer and analysed using EpiInfo 6 software (Centers for Disease Control, Atlanta, GA, USA; WHO, Geneva, Switzerland). The Chi-squared test or Fisher's exact test was used to compare proportions. The Chisquared test for linear trend was used to assess trends over time. Logistic regression was used for multivariate analysis.

\section{Results}

\section{Response rate}

During the 8 yrs of surveillance, the number of laboratories reporting cases to the NRC-RAD decreased from 363 to 338. The decrease was mainly due to discontinuation of mycobacterial activities by some laboratories or to fusion of laboratories. In all cases, the new laboratories resulting from these fusions participated in the network. The mean response rate to the questionnaire was $97 \%$ during the 8 -yr period (range 95-99\%). Missing data always originated from laboratories reporting less than five patients per year.

\section{Annual prevalence and incidence rates}

The number of MDRTB patients reported each year ranged 26-58 accounting for a total of 328 patients. The annual prevalence of MDRTB among the total number of patients with culture-positive TB ranged $0.4-0.9 \%$ (table 1 ).

A substantial proportion (mean 16\%) of the MDRTB patients were reported during several subsequent years, i.e.
Table 2. - Year of the first report of multidrug-resistant (MDR) tuberculosis patients, 1992-1999

\begin{tabular}{lccccccccc}
\hline \multirow{2}{*}{$\begin{array}{c}\text { Year } \\
\text { Total MDR } \\
\text { patients } n\end{array}$} & \multicolumn{7}{c}{ Cases reported for the first time n } \\
\cline { 3 - 9 } & & 1992 & 1993 & 1994 & 1995 & 1996 & 1997 & 1998 & 1999 \\
\hline 1992 & 48 & 48 & & & & & & & \\
1993 & 40 & 7 & 33 & & & & & & \\
1994 & 58 & 8 & 6 & 44 & & & & & \\
1995 & 40 & 3 & 7 & 4 & 26 & & & & \\
1996 & 29 & 1 & 0 & 3 & 3 & 22 & & & \\
1997 & 26 & 2 & 1 & 1 & 0 & 4 & 18 & & \\
1998 & 39 & 1 & 0 & 2 & 1 & 1 & 3 & 31 & \\
1999 & 48 & 1 & 0 & 0 & 1 & 0 & 0 & 2 & 44 \\
Total & 328 & & & & & & & & \\
\hline
\end{tabular}

chronic patients (table 2). Consequently, the total number of distinct patients with MDRTB was 264 during the $8-y r$ surveillance period and the mean annual incidence rate (distinct patients) was $<0.5 \%$. At least one case of MDRTB was reported in 22 of the 23 regions of France (including the overseas countries) during the 8-yr period. One-half of the 264 patients were reported in the Paris area (Ile de France region), compared with nine other regions reporting one or two cases each.

\section{Characteristics of multidrug-resistant tuberculosis cases}

The characteristics of the 264 distinct MDRTB patients reported during the 8 -yr surveillance period are shown in table 3 . The majority of patients were male $(69.7 \%)$, and more than one-half were foreign-born $(55.7 \%)$. Among the latter group, 27.9\% were born in Northern Africa, 37.4\% in subSaharan Africa, $16.3 \%$ in Asia, 5.5\% in Southern America, and $12.9 \%$ in Europe. Moreover, $30.6 \%$ of foreign-born patients came to France especially for management of their MDRTB. Patients aged 25-44 yrs represented $54.9 \%$ of all cases and HIV-coinfected patients represented 20.8\%. HIV coinfection was more frequent among males $(\mathrm{p}=0.02)$, but not more frequent among foreign-born patients $(\mathrm{p}=0.69)$. Foreignborn patients were younger than French-born patients (median age 34 yrs versus 49 yrs, respectively, $\mathrm{p}<0.01$ ). However, there was no difference regarding sex.

The site of TB was only pulmonary in $78.4 \%$ of the cases, only extrapulmonary in $7.2 \%$ and both pulmonary and extrapulmonary in $14.4 \%$. Extrapulmonary TB, either alone or associated with pulmonary TB, was associated with HIV coinfection ( $45 \%$ in HIV-coinfected and $15 \%$ in non-HIVcoinfected patients, $\mathrm{p}<0.01)$. The smear result was positive in $59.1 \%$ of the patients. Smear-positive results were slightly more frequent among HIV-coinfected patients (70\% versus $60 \%$ ) but the difference was not statistically significant $(\mathrm{p}=0.21)$.

Considering the first-line drugs (INH, RMP, EMB and $\mathrm{SM}), 37.9 \%$ of the MDR strains were resistant only to INH

Table 1.- Number of multidrug-resistant (MDR) tuberculosis (TB) and culture-positive TB cases, 1992-1999

\begin{tabular}{lcccccccc}
\hline & \multicolumn{8}{c}{ Year of report } \\
\cline { 2 - 9 } & 1992 & 1993 & 1994 & 1995 & 1996 & 1997 & 1998 \\
\hline MDR & 48 & 40 & 58 & 40 & 29 & 26 & 39 & 49 \\
Total culture-positive & 8441 & 8539 & 7751 & 7119 & 6441 & 5917 & 5766 & 5597 \\
MDR prevalence & $0.6(0.4-0.7)$ & $0.5(0.3-0.6)$ & $0.7(0.5-0.9)$ & $0.6(0.4-0.8)$ & $0.5(0.3-0.6)$ & $0.4(0.3-0.6)$ & $0.7(0.5-0.9)$ & $0.9(0.6-1.1)$ \\
\hline
\end{tabular}

Data are presented as $\mathrm{n}$ or $\%(95 \%$ confidence interval). 
Table 3. - Characteristics of the 264 patients with multidrug-resistant tuberculosis reported from 1992-1999

\begin{tabular}{|c|c|c|c|c|c|}
\hline \multirow[t]{2}{*}{ Characteristic } & \multirow[t]{2}{*}{ Total } & \multicolumn{2}{|c|}{ Previously treated patient } & \multicolumn{2}{|c|}{ Patient reported more than once } \\
\hline & & Yes & No & Yes & No \\
\hline Total cases & $264(100)$ & $174(100)$ & $88(100)$ & $42(100)$ & $222(100)$ \\
\hline $15-24$ yrs & $20(7.6)$ & $12(6.9)$ & $8(9.1)$ & $3(7.1)$ & $17(7.7)$ \\
\hline $25-34$ yrs & $78(29.5)$ & $44(25.3)$ & $34(38.6)$ & $11(26.2)$ & $67(30.2)$ \\
\hline $35-44$ yrs & $67(25.4)$ & $46(26.4)$ & $20(22.7)$ & $13(31.0)$ & $54(24.3)$ \\
\hline $45-64$ yrs & $61(23.1)$ & $44(25.3)$ & $16(18.2)$ & $12(28.6)$ & $49(22.1)$ \\
\hline$\geqslant 65 \mathrm{yrs}$ & $38(14.4)$ & $28(16.1)$ & $10(11.4)$ & $3(7.1)$ & $35(15.8)$ \\
\hline Male & 184 (69.7) & $128(73.6)$ & $54(61.4)$ & $26(61.9)$ & $158(71.2)$ \\
\hline Female & $80(30.3)$ & $46(26.4)$ & 34 (38.6) & $16(38.1)$ & $64(28.8)$ \\
\hline \multicolumn{6}{|l|}{ Country of birth } \\
\hline France & 115 (43.6) & $76(43.7)$ & $39(44.3)$ & $14(33.3)$ & $101(45.5)$ \\
\hline Other country & 147 (55.7) & $96(55.2)$ & $49(55.7)$ & $28(66.7)$ & 119 (53.6) \\
\hline Unknown & $2(0.8)$ & $2(1.1)$ & $0(0.0)$ & $0(0.0)$ & $2(0.9)$ \\
\hline \multicolumn{6}{|l|}{ Place of residence } \\
\hline France & $218(82.6)$ & $142(81.6)$ & $76(86.4)$ & $36(85.7)$ & $182(82.0)$ \\
\hline Other country & $45(17.0)$ & $31(17.8)$ & $12(13.6)$ & $6(14.3)$ & $39(17.5)$ \\
\hline Unknown & $1(0.4)$ & $1(0.6)$ & $0(0.0)$ & $0(0.0)$ & $1(0.5)$ \\
\hline HIV-positive & $55(20.8)$ & $29(16.7)$ & $24(27.3)$ & $5(11.9)$ & $50(22.5)$ \\
\hline HIV-negative & 169 (62.9) & $114(65.5)$ & $55(62.5)$ & 33 (78.6) & $136(61.3)$ \\
\hline HIV unknown & $40(16.3)$ & $31(17.8)$ & $9(10.2)$ & $4(9.5)$ & $36(16.2)$ \\
\hline \multicolumn{6}{|l|}{ Site of disease } \\
\hline Pulmonary & 207 (78.4) & $144(82.8)$ & $61(69.3)$ & $36(85.7)$ & $171(77.0)$ \\
\hline Extrapulmonary & $19(7.2)$ & $10(5.7)$ & $9(10.2)$ & $5(11.9)$ & 33 (14.9) \\
\hline Both & $38(14.4)$ & $20(11.5)$ & $18(20.5)$ & $1(2.4)$ & $18(8.1)$ \\
\hline \multicolumn{6}{|l|}{ Microscopy } \\
\hline Positive & $156(59.1)$ & $110(63.2)$ & $45(51.1)$ & $29(69.0)$ & $127(57.2)$ \\
\hline Negative & 94 (35.6) & $52(29.9)$ & $41(46.6)$ & $10(23.8)$ & $84(37.8)$ \\
\hline Unknown & $14(5.3)$ & $12(6.9)$ & $2(2.3)$ & $3(7.1)$ & $11(5.0)$ \\
\hline \multicolumn{6}{|c|}{ Resistance to other first-line drugs } \\
\hline None & $100(37.9)$ & $70(40.2)$ & $30(34.1)$ & $15(35.7)$ & $85(38.3)$ \\
\hline SM alone & 78 (29.5) & $50(28.7)$ & $28(31.8)$ & $10(23.8)$ & $68(30.6)$ \\
\hline EMB alone & $18(6.8)$ & $10(5.7)$ & $8(9.1)$ & $4(9.5)$ & $14(6.3)$ \\
\hline SM and EMB & $68(25.8)$ & $44(25.3)$ & $22(25.0)$ & $13(31.0)$ & $55(24.8)$ \\
\hline
\end{tabular}

Data are presented as $\mathrm{n}(\%) .{ }^{\#}$ : patient reported more than once (at least for 2 yrs) during the 1994-1998 period. All information were missing for one case in 1999 and two cases had no information on history of treatment. HIV: human immunodeficiency virus; SM: streptomycin; EMB: ethambutol.

and RMP. In addition, $29.5 \%$ of the strains were resistant to SM, $6.8 \%$ to EMB, and $25.8 \%$ to both SM and EMB. Resistance to both SM and EMB was less frequent among French-born patients than among foreign-born patients $(29 \%$ versus $50 \%, \mathrm{p}<0.01)$, even after stratification of previous history of treatment.

The results of the in vitro susceptibility tests were considered separately for pyrazinamide because of uncertainties of interpretation [12]. Indeed, unequivocal results were available for only 154 strains, $39(25.3 \%)$ being reported as resistant.

Susceptibility data to second-line drugs were not reported for all strains. Susceptibility rates to these drugs were $89.6 \%$ to kanamycin or amikacin (112 of 125 strains), 94.1 to capreomycin (96 of 102 strains), 67.5\% to thioamides (81 of 120 strains), $90.5 \%$ to fluoroquinolones (either ofloxacin or sparfloxacin, 114 of 126 strains), 64\% to tiacetazone (64 of 100 strains), $95.7 \%$ to cycloserine (111 of 116 strains), and 92.2 to para-aminosalicylic acid (94 of 102 strains).

Among the 264 patients, 174 (65.9\%) had been previously treated, and $88(33.3 \%)$ were classified as new patients (previous history of treatment was unknown for two patients). Compared with new patients, previously treated patients were more likely to be male $(73.6 \%$ versus $61.4 \%, \mathrm{p}=0.04)$, to have only pulmonary TB $(82.8 \%$ versus $69.3 \%, \mathrm{p}=0.02)$, and to be smear-positive $(63.2 \%$ versus $51.1 \%, \mathrm{p}=0.02)$. In addition, previously treated patients were slightly older than new patients (median age 39 yrs versus 35 yrs, $\mathrm{p}=0.03$ ). In contrast, new patients were more likely to be HIV coinfected $(27.3 \%$ versus $16.7 \%, \mathrm{p}=0.09$ ). New and previously treated patients were not statistically different regarding foreign-born status and place of residence.

\section{Multivariate analysis}

Country of birth, age, sex, HIV coinfection, site of TB and smear result were introduced in a backward logistic regression model to assess characteristics independently associated to primary or secondary multidrug resistance. HIV coinfection (odds ratio (OR) 2.02, 95\% confidence interval 1.04-3.95) and female status $(2.01,1.12-3.62)$ were statistically associated with primary resistance. On the contrary, a smear-positive result was associated with secondary resistance $(0.50,0.29-0.86)$.

In a second multivariate model assessing characteristics independently associated with the risk of being reported more than once to the NRC-RAD (chronic status), foreign-birth (1.91, 0.91-3.99) and a smear-positive result at MDR diagnosis $(1.98,0.91-4.30)$ remained associated with chronic status, but the association was not statistically significant ( $\mathrm{p}=0.08$ for both).

\section{Trend over time}

There was no significant linear trend over time when considering the overall proportion of MDRTB patients reported each year, the proportion of HIV-coinfected patients, and the 
proportion of foreign-born patients. However, from 19931999, there was a significant increase in the proportion of patients reported for the first time to the NRC-RAD $(p=0.03)$, in the number of new patients, i.e. those with no previous history of treatment $(\mathrm{p}=0.002)$, and in the proportion of foreign patients coming to France for the management of their MDRTB (permanent residence outside France; $\mathrm{p}=0.02$ ). There was no significant increase in the annual number of patients originating from Eastern Europe.

\section{Discussion}

The results of the ongoing surveillance of MDRTB in France between 1992-1999 shows that a laboratory-based surveillance system obtaining data recommended by the WHO and International Union Against Tuberculosis and Lung Disease yields high added value. First, the system allowed the measurement of the magnitude of the problem and the evaluation of trends over time; the prevalence of MDRTB remains low in France $(<1 \%)$. Secondly, it acquired data that allows the evaluation of the quality of the TB programme; the fact that $16 \%$ of the patients were reported at least twice suggests that they were not cured. Thirdly, it confirmed that foreign-born patients were at higher risk of MDRTB and drew attention on the recent trend toward an increase in the proportion of foreign-patients coming to France to be treated. Finally, and as expected, it showed that HIV coinfection is related to MDRTB, but that it is true in France only for patients with no history of treatment (OR 2.0).

The surveillance of MDRTB in France complies with most recommended standards, although the system was established before international recommendations for standardisation of drug resistance surveillance in Europe [13]. These results show that, in countries where anti-TB drug resistance surveillance is not linked to the TB case notification system, a system based on a national network of laboratories provides useful data. Such a network allows the reliable assessment of trends over time, as proven by its stability over the 8 -yr period. Indeed, in order to ensure reliable data over time, it is important to use personal identifiers, since chronic patients may be reported by several laboratories over many years. In addition, changes in network members over time have to be carefully identified in order to ensure completeness of data. Of interest, the trend in the total number of cases with culture-positive TB reported to the network was similar to the trend of cases reported to the mandatory TB case notification system, with a mean decrease of $8.5 \%$ per year from 1993-1997 followed by a decrease of $3 \%$ per year compared with $9.2 \%$ and $1.5 \%$, respectively. Since the source of the data is unrelated to the mandatory TB case notification system organised in France, and although it was not designed for this, the surveillance system described here could be used to assess completeness of both systems by techniques like capture-recapture.

This laboratory-based surveillance demonstrated that the size of the MDRTB problem is low and remains stable in France. The prevalence of MDRTB in France of $<1 \%$ is comparable with figures reported in most Western European countries except Germany (prevalence 1.4\%), Greece $(5.1 \%$ ) and Italy $(6.3 \%)$ [14]. The low and stable number of MDRTB cases suggests that the global management of TB in France is satisfactory because very few cases of MDRTB are created each year. Indeed, no trends were observed with regards to the prevalence or incidence of MDRTB. However, the overall stable trend may be falsely reassuring. Indeed, in-depth analysis of data using personal identifiers pointed to a rather worrying proportion of chronic carriers. One epidemiological consideration could be made concerning the state of chronic carriers of MDR bacilli. If the pathogenicity of MDR strains were similar to that of fully susceptible strains, the number of primary MDRTB cases would increase in France and in other industrialised countries due to persistent exposure of the population to chronic carriers. It is not the case so far and a recent study may bring some light on this phenomenon [15]. Indeed, this study showed that nonimmunosuppressed patients harbouring INH-resistant strains (including MDR strains) are less likely to create clusters than patients harbouring INH-susceptible strains. However, this does not apply to immunosuppressed patients. This is in accordance with the association of HIV coinfection and MDRTB found for the new patients in the present study.

There was a shift toward a higher proportion of foreignborn patients in the recent years of the surveillance. In France, most of these originated from Africa. To date, an increase in the number of patients coming from Eastern Europe was not observed despite the political changes in this region in the 1990s, but such findings may vary from country to country [16]. Since MDRTB prevalence is worrying in some Eastern European countries, particular attention should be paid to this issue in the near future. The recent extension of the European Union towards the east may facilitate patient exchange and surveillance systems, which can alert health authorities on moving trends.

Results of molecular fingerprinting of a subset of MDR strains isolated in France has been previously published and few clusters were identified $[17,18]$. This is not surprising according to the low number of MDRTB patients managed in France, their multiple geographic origins and diverse backgrounds. However, systematic molecular typing of MDR strains continues in France in order to compare results with the international MDR database.

The present surveillance system was not designed to monitor outcome of MDR patients. However, it brings two useful pieces of information together regarding the treatment of MDRTB in France. First, since a high proportion of strains are still susceptible to second-line drugs, antituberculosis drug regimens would be expected to cure the patients if properly designed and administered following WHO recommendations [19]. Indeed, following the WHO list of secondline drugs ordered by antibacterial activity, $70 \%$ of the MDR strains are still susceptible to amikacin, fluoroquinolones, pyrazinamide and EMB. Secondly, the cure rate may not be satisfactory because there are chronic cases reported over many consecutive years. Therefore, using the database, specific studies were performed focusing on outcome. The outcome of MDRTB patients reported to the NRC-RAD in 1994 were evaluated by a retrospective study. The results confirmed that the management of MDRTB patients was not optimal and that patient outcome was not satisfactory [3]. MDRTB patients were managed by $>40$ different sites disseminated throughout the territory. The relatively low number of patients managed each year in each centre precluded microbiological laboratories and clinicians to develop expertise in the management of these difficult-to-treat patients. Consequently, as recommended by the WHO and already implemented in some countries, one or a few reference teams working in collaboration with national associations of physicians and microbiologists should be established in France in order to improve the outcome of MDRTB patients. This is especially important in the prospect of the increase in the number of MDRTB patients coming to France and Europe from high prevalence areas.

The need for management and follow-up of multidrugresistant tuberculosis patients by reference team(s) has been presented to the National Association Of Respiratory Physicians [20]. These recommendations are currently being implemented 
in France and the first results are encouraging [21]. A decrease in chronic cases reported by the laboratory-based surveillance system and specific surveys assessing multidrug-resistant tuberculosis outcome will evaluate the impact of the implementation of these recommendations.

\begin{abstract}
Acknowledgements. The authors are grateful to the microbiologists of the network for their active and ongoing participation in the collection of data, and without whom the surveillance would have been impossible. They are also indebted to J. Grosset who initiated the surveillance of multidrug-resistant tuberculosis in France. They would also like to thank A-C. de Benoist, B. Decludt, S. Haeghebaert and V. Schwoebel, former members of the National Reference Centre for their active participation to the surveillance.
\end{abstract}

\section{References}

1. Espinal MA, Laszlo A, Simonsen L, et al. Global trends in drug resistance to antituberculosis drugs. $N$ Engl J Med 2001; 344: 1294-1303.

2. Mitchison DA, Nunn A. Influence of initial drug resistance on the response to short-course chemotherapy of pulmonary tuberculosis. Am Rev Respir Dis 1986; 133: 423-430.

3. Flament-Saillour M, Robert J, Jarlier V, Grosset J. Outcome of multidrug-resistant tuberculosis in France: a nationwide case-control study. Am J Respir Crit Care Med 1999; 166: 587-593.

4. World Health Organization. Global tuberculosis control. WHO Report 2001. www.who.int/gtb/publications/globrep01/ index.html. World Health Organization, WHO/CDS/TB/ 2001.287, 2001. Last updated: July 15, 2003

5. Beck-Sagué C, Dooley SW, Hutton MD, et al. Hospital outbreak of multidrug-resistant Mycobacterium tuberculosis infections. Factors in transmission to staff and HIV-infected patients. JAMA 1992; 268: 1280-1286.

6. Bouvet E, Casalino E, Mendoza-Sassi G, et al. A nosocomial outbreak of multidrug-resistant Mycobacterium bovis among HIV-infected patients. A case-control study. AIDS 1993; 7: 1453-1460.

7. Fischl MA, Uttamchandani RB, Daikos GL, et al. An outbreak of tuberculosis caused by multiple-drug-resistant tubercle bacilli among patients with HIV infection. Ann Intern Med 1992; 117: 177-183.

8. Frieden TR, Sherman LF, Maw KL, et al. A multiinstitutional outbreak of highly drug-resistant tuberculosis. Epidemiology and clinical outcomes. JAMA 1996; 276: 1229-1235.

9. Comstock GW, Cauthen GM. Epidemiology of tuberculosis. In: Reichmann LB, Hershfield ES, eds. Tuberculosis, a
Comprehensive International Approach. New York, Marcel Dekker, 1993; pp. 23-48.

10. Canetti G, Gay P, Le Lirzin M. Trends in the prevalence of primary drug resistance in pulmonary tuberculosis in France from 1962 to 1970: a national survey. Tubercle 1972; 53: 5783.

11. World Health Organization Geneva/International Union Against Tuberculosis and Lung Disease Paris. Guidelines for surveillance of drug resistance in tuberculosis. Int J Tuberc Lung Dis 1998; 2: 72-89.

12. Canetti G, Fox W, Khomenko A, et al. Advances in techniques of testing mycobacterial drug sensitivity, and the use of sensitivity tests in tuberculosis control programmes. Bull World Health Organ 1969; 41: 21-43.

13. Veen J, Raviglione $\mathrm{M}$, Rieder $\mathrm{H}$, et al. Standardized tuberculosis treatment outcome monitoring in Europe. Recommendations of a Working Group of the World Health Organization (WHO) and the European Region of the International Union Against Tuberculosis and Lung Disease (IUATLD) for uniform reporting by cohort analysis of treatment outcome in tuberculosis patients. Eur Respir $J$ 1998; 12: 505-510.

14. Institut de Veille Santaire/European Commission and the national coordinators for tuberculosis surveillance in the World Health Organization European region. EuroTB. Surveillance of tuberculosis in Europe. Report on tuberculosis case notified in 1999. http://www.eurotb.org. 2001.

15. Burgos M, DeRiemer K, Small P, Hopewell P, Daley C. Relative fitness of drug-resistant and drug-susceptible strains of Mycobacterium tuberculosis in San Francisco. Am J Respir Crit Care Med 2002; 165: A18.

16. Loddenkemper R. The need for antituberculosis drug resistance surveillance in Europe. Eur Respir $J$ 2000; 16: 195-196.

17. Grosset J, Trystram D, de Benoist A-C, et al. Surveillance de la tuberculose à bacilles multirésistants en France en 1995. [Surveillance of multidrug-resistant tuberculosis in France, 1995]. Bull Epid Hebdo 1998; 13: 53-54.

18. Gutierrez MC, Vincent V. Contribution du typage moléculaire à la surveillance de la tuberculose multirésistante en France, 1995-2000. [Contribution of molecular typing to multidrug-resistant tuberculosis in France, 1995-2000]. Bull Epid Hebdo 2002; 10/11: 73-75.

19. Crofton J, Chauley P, Maher D. Guidelines for the Management of Drug-Resistant Tuberculosis. Geneva, World Health Organization, 1997.

20. Robert J, Jarlier V. La tuberculose multirésistante en France. Rev Mal Respir 2002; 19: 21-23.

21. Uffredi ML, Robert J, Truffot-Pernot C, Trystram D, Jarlier V, Grosset J. Multidrug-resistant tuberculosis (MDR-TB): prospective study on effectiveness of individually tailored regimens (DOTS-plus strategy). 30th IUATLD World Conference on Lung Health, 1999, Madrid, Spain. Int J Tuberc Lung Dis 1999; 3: Suppl. 1, S83. 